\title{
Is transthoracic lung biopsy a safe procedure?
}

\author{
Cezar Iulian Bețianu ${ }^{1}$, Mihaela Goicea ${ }^{1}$, Giorgiana Ion ${ }^{1}$, Andrei Bondari²
}

${ }^{1}$ Department of Radiology and Medical Imaging, „Carol Davila” Central Military Emergency University Hospital, Bucharest, ${ }^{2}$ Radiology and Imaging Department, Faculty of Medicine, University of Medicine and Pharmacy, Craiova, Romania

\section{To the Editor,}

The transthoracic lung biopsy is a minimally invasive diagnostic procedure used for determining the management of patients with a high suspicion for malignancy lesions. Although the importance of percutaneous lung biopsy has been clearly stated by many studies [1-3], we observed that percutaneous biopsy is still regarded with great concern by both patients and physicians mostly because of its possible complications. In literature, complication rates after percutaneous lung biopsies reach up to $38.8 \%$ while major complications occur in up to $5.7 \%$ of cases [3]. The most frequently described post-procedural complication is pneumothorax, occurring in $25 \%$ of cases [3]. Severe complications include pneumothorax requiring intervention, haemothorax, air embolism, needle track seeding and death [4]. Literature describes a 0.061 $\%$ rate for air embolism and tumor seeding, $0.092 \%$ rate for haemothorax and $0.07-0.15 \%$ rate for death [4]. The purpose of this letter is to point out that percutaneous lung biopsy is underappreciated since it is a rapid, accurate and safe diagnostic tool with a relatively low rate of complications.

A group of 317 patients underwent percutaneous lung core biopsies in our hospital between July 2015 and June 2018. The procedures were guided either by computed tomography (CT) or ultrasound (US) and were performed with $18 \mathrm{G}$ Tru-cut needles. The decision for performing the biopsy as well as the selection of the puncture site

Received 17.12.2019 Accepted 09.01.2020

Med Ultrason

2020, Vol. 22, No 1, 114-115, DOI: 10.11152/mu-2360,

Corresponding author: Giorgiana Ion

„Carol Davila” Central Military Emergency

University Hospital, Bucharest, Romania

134 Calea Plevnei, Bucharest 010825, Romania

E-mail: pavaloiu.giorgiana@gmail.com

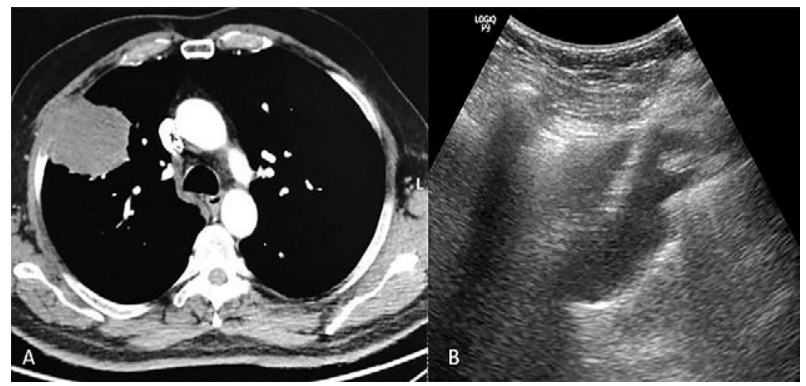

Fig 1. A. Axial CT image shows a pulmonary tumour of 70/80 $\mathrm{mm}$ diameter located in the superior right lobe with chest wall and rib invasion. B. Ultrasound-guided transthoracic biopsy of the tumour. No postprocedural complications occurred.

were made based on CT examinations; this was considered sufficient also for subsequently US-guided procedures, since the morphologic correlation between CT and US in lung lesions is reliable [5]. Of the 317 patients, complications occurred in 33 cases (10.41\%): 25 pneumothoraces $(7.89 \%), 3$ haemothoraces $(0.95 \%), 7$ alveolar haemorrhages (2.21\%) and 1 infection at puncture site $(0.32 \%)$, in a patient with decompensated diabetes mellites. There were no cases of air embolism, needle track seeding or death.

To sum up, the lung biopsy is a relatively safe procedure, given the adequate selection of patients. The complication rates in our hospital are comparable to larger internationally published data.

\section{References}

1. Galluzzo A, Genova C, Dioguardi S, Midiri M, Cajozzo M. Current role of computed tomography-guided transthoracic needle biopsy of metastatic lung lesions. Future Oncol 2015;11(2 suppl):43-44.

2. Russo U, Sabatino V, Nizzoli R, et al. Transthoracic computed tomography-guided lung biopsy in the new era of personalized medicine. Future Oncol 2019;15:1125-1134. 
3. Heerink WJ, de Bock GH, de Jonge GJ, Groen HJ, Vliegenthart R, Oudkerk M. Complication rates of CTguided transthoracic lung biopsy: meta-analysis. Eur Radiol 2017;27:138-148.

4. Tomiyama N, Yasuhara Y, Nakajima Y, et al. CT-guided needle biopsy of lung lesions: A survey of severe com- plication based on 9783 biopsies in Japan. Eur J Radiol 2006;59:60-64.

5. Chira R, Chira A, Mircea PA. Intrathoracic tumors in contact with the chest wall - ultrasonographic and computed tomography comparative evaluation. Med Ultrason 2012;14:115-119.

\title{
Suprastyloid crest of the radius in wrist ultrasonography: a bony prominence not to mistake for Lister's tubercle
}

\author{
Mathieu Boudier-Revéret ${ }^{1}$, Stéphanie Jean ${ }^{1}$, Chueh Hung Wu ${ }^{2}$
}

${ }^{1}$ Department of Physical Medicine and Rehabilitation, Centre Hospitalier de l'Université de Montréal, Montreal, Canada, ${ }^{2}$ Department of Physical Medicine and Rehabilitation, National Taiwan University Hospital, College of Medicine, National Taiwan University, Taipei, Taiwan

\section{To the Editor,}

Ultrasound (US) is becoming increasingly popular amongst physiatrists to diagnose and treat musculoskeletal (MSK) pathologies. Well-designed standardized protocols have been published to scan the most common painful joints and surrounding structures. At the wrist, Lister's tubercle, which is also known as the dorsal tubercle, serves as the main landmark to identify the dorsal extensor compartments at the distal radius [1]. It is a clear boundary between the $2^{\text {nd }}$ compartment, which sits on the radial side of the tubercle, and the $3^{\text {rd }}$ and $4^{\text {th }}$ compartments, which sit on its ulnar side. However, in many patients, another similarly shaped bony prominence is present between the $1^{\text {st }}$ and $2^{\text {nd }}$ compartments. It is not mentioned in most reference MSK US textbooks $[2,3]$ and is called the suprastyloid crest of the radius (fig 1 , supplementary video 1 , on the journal site). Its Latin name is crista suprastyloidea radii [4] and it lies just proximal to the radial styloid and corresponds to the actual insertion site of the brachioradialis muscle.

Received 02.01.2020 Accepted 05.01.2020

Med Ultrason

2020, Vol. 22, No 1, 115-116, DOI: 10.11152/mu-2395,

Corresponding author: Dr. Boudier-Revéret MD

Hôtel-Dieu du Centre Hospitalier de l'Université de Montréal, 3840, Saint-Urbain St., Montreal, QC, H2W 1T8, Canada

Phone: 1-514-890-8000

E-mail: mathieu.boudier-reveret@umontreal.ca

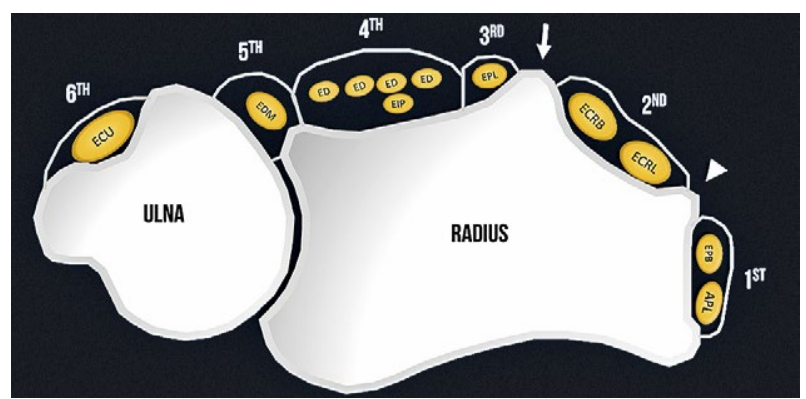

Fig 1. This schematic diagram depicts the anatomy of the six extensor compartments at the dorsal wrist. Lister's tubercle, also known as the dorsal tubercle, (arrow) divides the $2^{\text {nd }}$ and $3^{\text {rd }}$ compartments, whereas the suprastyloid crest (arrowhead) divides the $1^{\text {st }}$ and $2^{\text {nd }}$ compartments. $1^{\text {st }}$ compartment: APL, abductor pollicis longus. EPB, extensor pollicis brevis. $2^{\text {nd }}$ compartment: ECRL, extensor carpi radialis longus. ECRB, Extensor carpi radialis brevis. $3^{\text {rd }}$ compartment: EPL, extensor pollicis longus. $4^{\text {th }}$ compartment: ED, extensor digitorum. EIP, extensor indicis proprius. $5^{\text {th }}$ compartment: EDM, extensor digiti minimi. $6^{\text {th }}$ compartment: ECU, extensor carpi ulnaris.

In some patients with a more prominent suprastyloid crest, it might be mistaken for Lister's tubercle by novice sonographers. In that case, the $1^{\text {st }}$ compartment would be misidentified as the $2^{\text {nd }}$ compartment, and confusion might ensue. However, no $3^{\text {rd }}$ compartment would be seen ulnar to the bony prominence, and the $2^{\text {nd }}$ compartment does not contain as many tendons as the $4^{\text {th }}$.

Easy ways to avoid this pitfall are to palpate the bony landmarks before placing the US probe on the patient's 
wrist. Lister's tubercle is located more dorsally on the radius, and the suprastyloid crest is more on the radial edge of the radius, even if the distance separating the two bony prominences is often around $1.5-2 \mathrm{~cm}$. Also, the ultrasonographer must use compartment anatomy to validate what compartment(s) he/she is looking at: How many tendons does it contain? What are the adjacent compartments? And of course: Where are the important bony landmarks?

Hopefully, this brief visual vignette will have educated readers on one of the less known bony prominences of the dorsal wrist, the suprastyloid crest, and will allow
US professors and students to better teach and learn sonographic anatomy.

\section{References}

1. Özçakar L, Kara M, Chang KV, et al. EURO-MUSCULUS/ USPRM Basic Scanning Protocols for wrist and hand. Eur J Phys Rehabil Med 2015;51:479-484.

2. Bianchi S, Martinoli C. Ultrasound of the musculoskeletal system. 2007: Springer Science \& Business Media.

3. Jacobson JA. Fundamentals of Musculoskeletal Ultrasound E-Book. 3rd Edition. Elsevier Health Sciences. 2017.

4. Platzer W. Zur Anatomie der "Sellabriicke" und ihre Beziehung. 1992.

\title{
Ultrasound imaging for lateral gastrocnemius muscle injury: tennis leg revisited
}

\author{
Yahya Doğan ${ }^{1}$, Levent Özçakar ${ }^{1}$
}

${ }^{1}$ Hacettepe University Medical School, Department of Physical and Rehabilitation Medicine, Ankara, Turkey

\section{To the Editor,}

A 37-year-old male presented with left calf pain for the last 5-6 months. He declared that the problem had suddenly occurred during a match (playing football) and that he had become a little better after rest. In the meantime, he had received a 3-week physical therapy after which he had partially improved. Medical history and physical examination was suggestive for 'tennis leg' (strain at the myotendinous junction of the medial head of the gastrocnemius muscle). Accordingly, ultrasound (US) examination was also performed. While US imaging was negative for 'tennis leg', diligent sonopalpation uncovered that the patient's complaints were actually localized more on the lateral side where the myotendinous junction appeared to be asymmetric as compared to the

Received 04.12.2019 Accepted 19.12.2019

Med Ultrason

2020, Vol. 22, No 1, 116-117, DOI: 10.11152/mu-2338,

Corresponding author: Yahya Doğan

Hacettepe University Medical School

Department of Physical and Rehabilitation

Medicine, Sthhiye, Ankara, Turkey

E-mail: yahyadogan111@hotmail.com

Fax number: +90 (312) 3105769

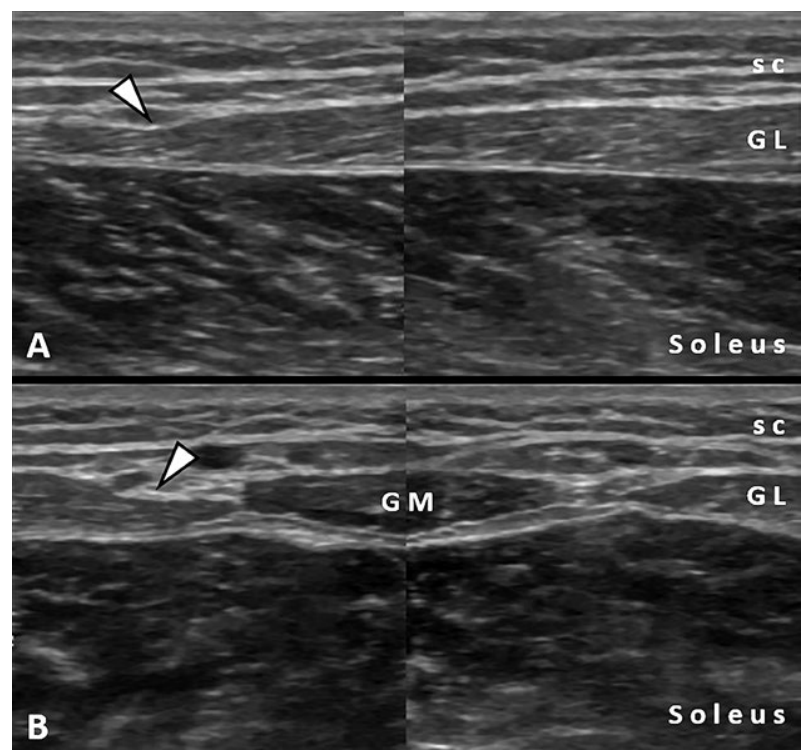

Fig 1. Comparative long- (A) and short-axis (B) US imaging for the calf muscles. White arrowhead shows the indentation at the myotendinous junction of the lateral head of the gastrocnemius muscle (left images) when compared to the normal side (right images). GM; Medial head of the gastrocnemius muscle, GL; Lateral head of the gastrocnemius muscle, sc; subcutaneous tissue. 
normal side (fig 1). In light of the US findings, the patient was diagnosed with a healing strain of the lateral head of the gastrocnemius muscle.

Calf pain is a common complaint among patients of all ages but is most frequent in young and active people (especially runners). Often, calf strains or ruptures are seen in the medial gastrocnemius muscle (i.e. 'tennis leg') but other components of the posterior leg including the lateral gastrocnemius, soleus and plantaris may also harbor the underlying cause/lesion [1]. These injuries usually occur during physical activities whereby forced dorsiflexion of the ankle and simultaneous extension of the knee are inevitable. Patients commonly report a sudden tear or 'pop' in their posterior leg after which they start suffering acute pain and tenderness [2]. According to the imaging studies, involvement of the medial gastrocnemius occurs in 58 to $65 \%$ of all cases; the lateral gastrocnemius in 8 to $38 \%$ and other muscles are less frequent $[2,3]$.

Presenting this (rare) case of ours, firstly, we emphasise that medical history and physical examination might sometimes be insufficient for an exact diagnosis. Secondly, sono-palpation is definitely helpful for demonstrating and the prompt understanding of the lesion (for the physician and for the patient alike) [4]. Last but not least, especially when an intervention is to be planned, the aforementioned 'sonographic understanding' will turn into 'precise targeting' also [5].

\section{References}

1. Bryan Dixon J. Gastrocnemius vs. soleus strain: how to differentiate and deal with calf muscle injuries. Curr Rev Musculoskelet Med 2009;2:74-77.

2. Fields KB, Rigby MD. Muscular calf injuries in runners. Curr Sports Med Rep 2016;15:320-324.

3. Koulouris G, Ting AYI, Jhamb A, Connell D, Kavanagh EC. Magnetic resonance imaging findings of injuries to the calf muscle complex. Skeletal Radiol 2007;36:921-927.

4. Çağlayan G, Özçakar L, Kaymak SU, Kaymak B, Tan AA. Effects of sono-feedback during aspiration of Baker's cysts: A controlled clinical trial. J Rehabil Med 2016;48:386-389.

5. Özçakar L (Ed). Sonographic atlas for common musculoskeletal pathologies. Milan: Edi.Ermes, 2017.

\title{
The utility of ultrasound examination in cubital tunnel syndrome caused by heterotopic ossification
}

\author{
Jakub Jačisko ${ }^{1}$, Karolína Sobotová ${ }^{1}, K^{\prime}$ amal Mezian²
}

${ }^{1}$ Department of Rehabilitation and Sports Medicine, Second Faculty of Medicine, Charles University and University Hospital Motol, ${ }^{2}$ Department of Rehabilitation Medicine, Charles University, First Faculty of Medicine and General University Hospital in Prague, Czech Republic

\section{To the Editor,}

A 65-year-old man presented with a one-year history of bilateral paresthesia of the ulnar side of the forearm and $4^{\text {th }}$ to $5^{\text {th }}$ finger, accompanied by numbness and weakness

Received 16.01.2020 Accepted 23.01.2020

Med Ultrason

2020, Vol. 22, No 1, 117-118, DOI: 10.11152/mu-2419,

Corresponding author: Jakub Jačisko, MD

Department of Rehabilitation and Sports

Medicine, Second Faculty of Medicine, Charles

University and University Hospital Motol,

V Úvalu 8415006 Prague, Czech Republic

Phone: 00420776246648

E-mail: jakub.jacisko@gmail.com of the mentioned area. The patient also reported sleep disturbance due to tingling sensations in his hand and fingers, resulting in awakening 3-4 times per night. His history involved 30 years working with a vibrating sander.

Clinical examination revealed hypotrophy of the interosseous muscles. Tinel's test was positive only over the ulnar nerve in the right ulnar sulcus area. Electromyography and nerve conduction studies revealed bilateral cubital tunnel syndrome, more severe on the right side. Ultrasound (US) examination of both elbows showed a hyperechoic mass causing acoustic shadowing, in close contact with the ulnar nerve on the right side. The ulnar nerve was swollen bilaterally (right side, cross-sectional area $15 \mathrm{~mm}^{2}$; left side $11 \mathrm{~mm}^{2}$ ). Radiographs of the 


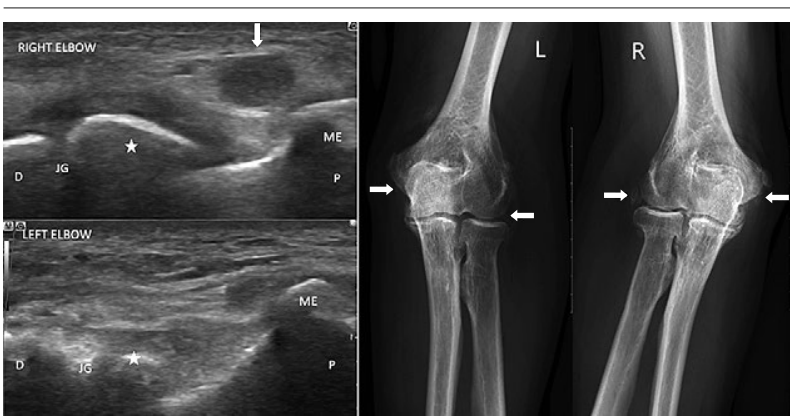

Fig 1. Left side: Ultrasound revealed a hyperechoic mass causing acoustic shadowing bilaterally, with close contact to a swollen ulnar nerve on the right side. $\mathrm{D}$, distal; $\mathrm{P}$, proximal; JG, joint gap; ME, medial epicondyle; white arrow, swollen ulnar nerve; asterisk, heterotopic ossification. Right side: Anteroposterior radiographs of both elbow joints. White arrows, heterotopic ossifications; L, left; R, right.

elbows showed multiple ossifications of the soft tissue around the medial and lateral humeral epicondyles bilaterally (fig 1). The patient was informed of the treatment options (corticosteroid injection/consideration of surgery) and opted for corticosteroid injection. The ulnar nerve on the right side was injected under US guidance with a mixture of $40 \mathrm{mg}(1 \mathrm{ml})$ methylprednisolone and $1 \mathrm{ml} \mathrm{1 \%}$ trimecaine. The injection provided satisfactory pain relief at the 2-week and 3-month follow-up.

The utility of US examination is found not only in diagnostic, but also in providing accurate therapy and fol- low-up. US examination can provide information about ulnar nerve morphology and surrounding structures that can be the cause of compression [1]. Therapeutic injection under US control is more precise and targeted. During follow-up, US examination can be a very useful tool for evaluating therapeutic effect - in this particular case, a reduction in the cross-sectional area of the swollen nerve.

Heterotopic ossification (HO) is a pathologic formation of bone within soft tissues. The main risk factors for $\mathrm{HO}$ are orthopedic surgery, trauma, brain or spinal cord injury and severe burns. Repetitive mechanical stress or microtrauma is generally thought to be present among the rest of the patients [2]. The long history of working with a vibrating sander in our patient was, probably, the risk factor for HO. More studies about HO in patients exposed to occupational vibration are needed.

Acknowledgment: This study was supported by the Foundation "Movement without Help", Prague, Czech Republic.

\section{References}

1. Kara M, Yalçın S, Yenigün D, Tiftik T, Malas FÜ, Özçakar L. Heterotopic ossification and cubital tunnel syndrome in traumatic brain injury: Ultrasound 'sees' both. J Back Musculoskelet Rehabil 2015;28:415-417.

2. Meyers C, Lisiecki J, Miller S, et al. Heterotopic Ossification: A Comprehensive Review. JBMR Plus. 2019;3:e10172.

\title{
Extensor digitorum brevis manus is uncommon but can easily be misinterpreted during wrist ultrasound examination
}

\author{
Ke-Vin Chang', Wei-Ting Wu' ${ }^{1}$, Ruei-Fang Wang², Levent Özçakar²
}

${ }^{1}$ Department of Physical Medicine and Rehabilitation, National Taiwan University Hospital, Bei Hu Branch and National Taiwan University College of Medicine, Taipei, Taiwan, ${ }^{2}$ Department of Emergency Medicine, Taipei City Hospital, Ranai Branch, Taipei, Taiwan, ${ }^{3}$ Department of Physical and Rehabilitation Medicine, Hacettepe University Medical School, Ankara, Turkey

Received 06.11.2019 Accepted 19.12.2019

Med Ultrason

2020, Vol. 22, No 1, 118-119, DOI: 10.11152/mu-2298,

Corresponding author: Ke-Vin Chang, $\mathrm{MD}, \mathrm{PhD}$

Department of Physical Medicine and

Rehabilitation, National Taiwan University

Hospital, Bei-Hu Branch and National Taiwan

University College of Medicine, Taipei, Taiwan

E-mail: kvchang011@gmail.com pattap@pchome.com.tw

\section{To the Editor}

A 37-year-old female complained of a distended sensation over her right wrist for the last six months. She reported occasional swelling at its dorsal aspect, accompanied by only soreness but no pain after 30 minutes of continuous computer use. The medical history was otherwise unremarkable. 
She was referred for an ultrasound (US) examination of the wrist. Pre-scanning physical examination revealed no tenderness and normal wrist motions. The transducer was first placed in the transverse plane at the ulnar styloid level. A hypoechoic mass was visualized between the extensor indicis (EI)and extensor digitorum communis tendons (fig 1A). Power Doppler imaging showed increased intralesional vascularity. Additionally, a ganglion cyst was seen emerging from the joint between the capitate and scaphoid. The transducer was then pivoted to the longitudinal plane and several parallel hyperechoic striates were seen inside the mass (fig 1B). We asked the patient to flex and extend the wrist and found out that the mass had a synchronized movement with finger extensor tendons (Supplementary Video 1, on the journal site). As a similar mass/structure was also identified at the contralateral wrist, she was considered to have bilateral extensor digitorum brevis manus (EDBM) muscles (fig 1C). We advised her to avoid any overuse of the wrist and gave instructions regarding the proper ergonomics for using electronic gadgets.

The EDBM is an accessory muscle inside the fourth extensor compartment of the dorsal wrist [1]. It usually emanates from the radiocarpal joint capsule and inserts distally on the EI tendon or the extensor hooks of middle, ring and little fingers. It receives innervation and vascular supply from the posterior interosseous nerve and artery, respectively. Its prevalence is around $2.3 \%$ in the general population based on a previous meta-analysis [2]. Participants with EDBM muscles are mostly asymptomatic and up to $50 \%$ of them have bilateral EDBM [1].

Of note, the existence of the EDBM muscle may increase the intra-compartmental pressure, leading to compression of the posterior interosseous nerve. In our case, concomitant presence of the EDBM muscle and the ganglion cyst further congested the compartment. Therefore, after repetitive wrist movements, the blood flow to the EDBM muscle increased, resulting in a distension feeling due to muscle edema.

Undoubtedly, US imaging is useful for the diagnosis of several wrist pathologies $[3,4]$. However, once unfamiliar with its presence, the EDBM muscle can easily/ likely be interpreted as a hypervascular tumor originating from the finger extensor tendon sheath- especially in the axial plane. During longitudinal imaging, its echotexture can be better clarified i.e. multiple hyperechoic lines un-

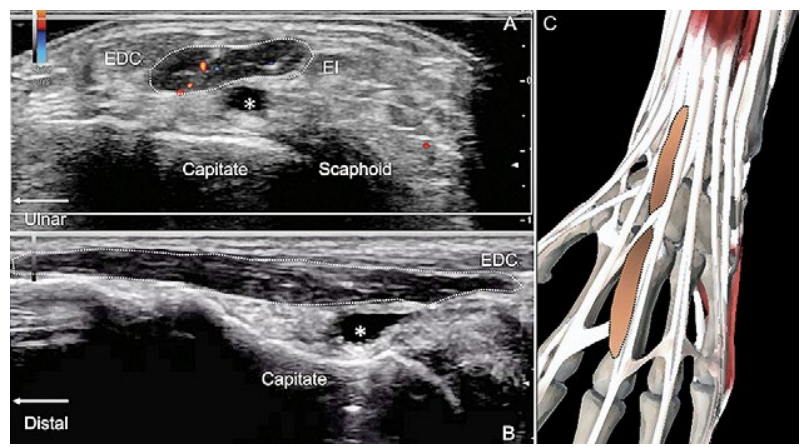

Fig 1. Ultrasound imaging of the extensor digitorum brevis manus muscle (white/black dashed area) in the transverse (A) and longitudinal (B) views. Schematic drawing (C). EDC, extensor digitorum communis tendon; EI, extensor indicis tendon; asterisks, ganglion cyst

der a hypoechoic background are consistent with perimysium in a muscle. Importantly, the synchronized movement with adjacent finger extensor tendons (on dynamic imaging) and bilateral presence (on comparative imaging) further confirmed the mass/structure to be an accessory muscle. In short, this report highlighted a relatively rare muscle variation on the dorsal hand, which should not be misinterpreted as a pathological finding during (a convenient but comprehensive) wrist US examination.

Acknowledgment: The present study is supported by the National Taiwan University Hospital, Bei-Hu Branch, Ministry of Science and Technology (MOST 106-2314-B-002-180-MY3) and Taiwan Society of Ultrasound in Medicine.

\section{References}

1. Shereen R, Loukas M, Tubbs RS. Extensor Digitorum Brevis Manus: A Comprehensive Review of this Variant Muscle of the Dorsal Hand. Cureus 2017;9:e1568.

2. Yammine K. The prevalence of extensor digitorum brevis manus and its variants in humans: a systematic review and meta-analysis. Surg Radiol Anat 2015;37:3-9.

3. Wu WT, Chang KV, Mezian K, et al. Ulnar Wrist Pain Revisited: Ultrasound Diagnosis and Guided Injection for Triangular Fibrocartilage Complex Injuries. J Clin Med 2019;8:E1540.

4. Chang KV, Wu WT, Özçakar L. Ultrasound Imaging for Dorsal Ulnar Cutaneous Neuropathy With Extensor Carpi Ulnaris Tendinopathy. Am J Phys Med Rehabil 2017;96:e191-e192. 